\title{
Phase diagram of the random Heisenberg antiferromagnetic spin-1 chain
}

\author{
Andreia Saguia \\ Centro Brasileiro de Pesquisas Físicas \\ Rua Dr. Xavier Sigaud 150 - Urca, \\ Rio de Janeiro, 22290-180, RJ - Brazil. \\ Beatriz Boechat and Mucio A. Continentino* \\ Departamento de Física - Universidade Federal Fluminense \\ Av. Litorânea s/n, Niterói, 24210-340, RJ - Brazil
}

(Dated: October 24, 2018)

\begin{abstract}
We present a new perturbative real space renormalization group (RG) to study random quantum spin chains and other one-dimensional disordered quantum systems. The method overcomes problems of the original approach which fails for quantum random chains with spins larger than $S=1 / 2$. Since it works even for weak disorder we are able to obtain the zero temperature phase diagram of the random antiferromagnetic Heisenberg spin-1 chain as a function of disorder. We find a random singlet phase for strong disorder and as disorder decreases, the system shows a crossover from a Griffiths to a disordered Haldane phase.
\end{abstract}

The study of the effects of disorder on quantum systems is an actual and important area of research [1-17. Intensive work on the last decades has deepened our understanding of the phase transitions which occur in pure quantum systems [18]. Now the main effort is concentrated in understanding the role of randomness in these transitions. This gives rise to new and interesting phenomena as the existence of Griffiths phases [2, 7]. In this connection random quantum spin chains have been intensive investigated. In the pure case their behavior is well known 19]. Also for spin-1/2 quantum antiferromagnetic chains a perturbative approach developed by Ma, Dasgupta and $\mathrm{Hu}(M D H)$ [1] and extended by Fisher [2] allows to obtain results which are essentially exact for this system. The picture which emerges for these chains is described by a random singlet phase $(R S P)$ where spins are coupled in pairs over arbitrary distances. In the renormalization group approach this random singlet phase is governed by an infinite randomness fixed point [2, 7, 17]. A straightforward extension of the $M D H$ method for biquadratic spin-1 chains has shown that in the Heisenberg case the perturbative RG approach may fail even for the case of strong disorder [3]. The reason is that in the elimination process of strong interactions, in which consists the $M D H$ approach, interactions stronger than those eliminated are generated. This failure is better demonstrated when the method is extended to finite temperatures where it gives rise to non-physical behavior as negative specific heat and so on [6]. Several proposals have been put forward to extend the $M D H$ method for quantum spin chains, with $S>1 / 2$ [11]-114, without undisputed success. The challenge in the case of quantum integer spin chains is particularly exciting as it deals with the question of the fate of the Haldane phase 20] in the presence of disorder. The existence of a gap in the excitation spectrum is not sufficient to guarantee the robustness of pure chain behavior with respect to the effects

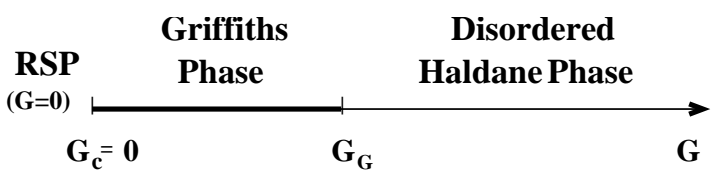

FIG. 1: The phase diagram of the spin-1 random Heisenberg antiferromagnetic chain. Disorder is inversely related to the gap $G$ of the initial rectangular distribution and $G_{G} \approx 0.45$.

of disorder. For gapped biquadratic chains, any amount of disorder drives the system to a random singlet phase or infinite randomness fixed point [3]. If this is not the case for Haldane chains there may be a unique property of integer chains which confers them a special stability with respect to the introduction of disorder. In fact Hida 16. using a density matrix renormalization group approach has not found any evidence for a $R S P$ for spin-1 chains even in the presence of strong disorder, although this is still a matter of controversy [12, 13].

In this Letter we propose a new approach to the spin-1 Heisenberg antiferromagnetic chain which is an improvement of the traditional $M D H$ perturbative renormalization group method. Our procedure avoids generation of interactions larger than those eliminated even for weak disorder. This provides a unique opportunity to obtain the phase diagram of the quantum, spin-1 Heisenberg chain as a function of disorder which is shown in Fig. 1. We consider here rectangular distributions of antiferromagnetic interactions $P(J)=(1 /(1-G)) \Theta(1-J) \Theta(J-$ $G)$. The gap $G$ is a measure of the amount of disorder being inversely related to it. We find for strong disorder, $G=0$, a random singlet phase. As $G$ increases, i.e., disorder decreases, there is a Griffiths phase characterized by exponents which depend on the distance to the infinite randomness fixed point at $G=0$. This Griffiths phase extends up to $G \approx 0.45$ and for weak disorder there is a disordered Haldane phase. 
a)

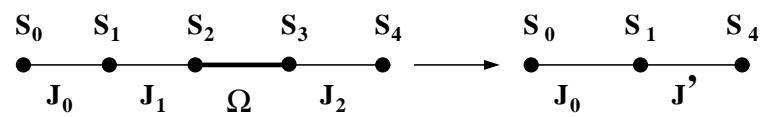

b)

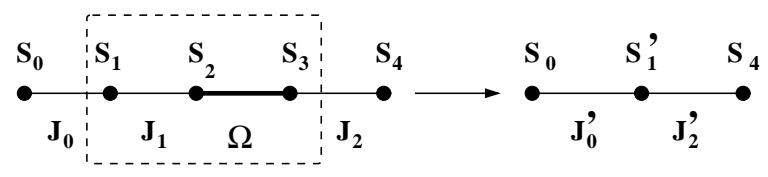

FIG. 2: The two elimination procedures as described in the $\operatorname{text}\left(J_{1}>J_{2}\right)$.

The $M D H$ method consists in finding the strongest interaction $(\Omega)$ between pairs of spins in the chain (see Fig.2a) and treating the coupling of this pair with their neighbors $\left(J_{1}\right.$ and $\left.J_{2}\right)$ as a perturbation. For a chain of spins $S=1$, after elimination of the strongest coupled pair, the new coupling between their neighbors is given by,

$$
J^{\prime}=\frac{4}{3} \frac{J_{1} J_{2}}{\Omega}
$$

The factor, $(4 / 3)>1$, in this equation is the source of the failure of perturbation theory. Let us assume, for example, that the largest of the neighboring couplings $\left(J_{1}, J_{2}\right)$ to the strongest interaction $\Omega$ in the chain is $J_{1}$. If $J_{1}>(3 / 4) \Omega$ than the new effective interaction $J^{\prime}$ is necessarily larger than one of those eliminated, in this case, than the weaker one $J_{2}$.

Our generalization of the $M D H$ method consists in either of the following procedures (Fig.1). If the largest neighboring interaction to $\Omega, J_{1}<(3 / 4) \Omega$, then we eliminate the strongest coupled pair obtaining an effective interaction between the neighbors to this pair which is given by Eq. 1. This effective interaction is always smaller than those eliminated.

Now suppose $J_{1}>(3 / 4) \Omega\left(J_{1}>J_{2}\right)$. In this case, we consider the trio of spins $S=1$ coupled by the two strongest interactions of the trio, $J_{1}$ and $\Omega$ and solve it exactly (see Fig. 2b). The ground state of this trio of spins $S=1$ is a degenerate triplet and it will be substituted by an effective spin-1 interacting with its neighbors through new renormalized interactions obtained by degenerate perturbation theory. This procedure which implies diagonalizing the $27 X 27$ matrix of the trio is carried out analytically. This is important for obtaining results on large chains and to deal with the large numbers of initial configurations that we use. These procedures guarantee that we always comply with the criterion of validity of perturbation theory and never, an interaction larger than those eliminated is generated [21] as shown in Fig. 3. Notice from this figure that even for the strong disorder case, with no gap in the original distribution of exchange couplings, the simple $M D H$ procedure fails.

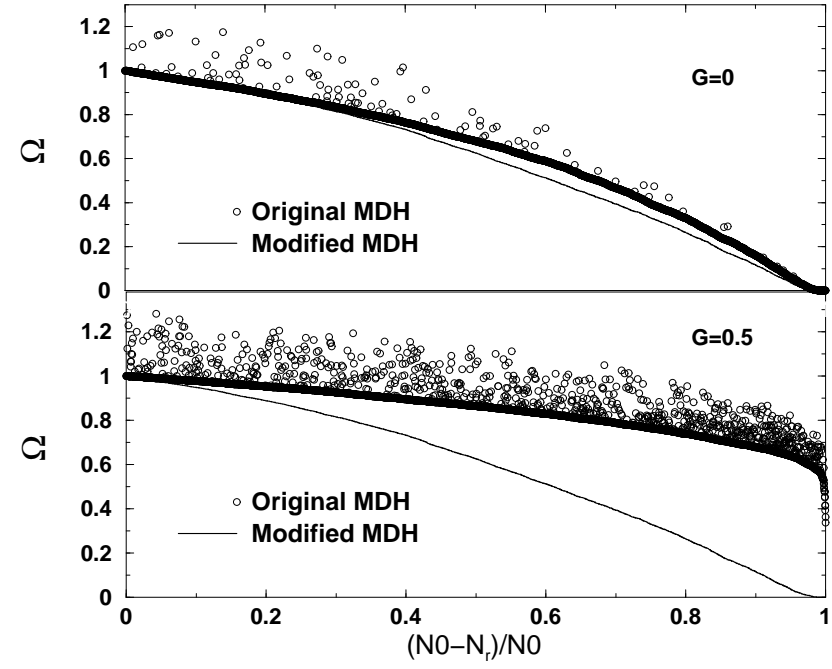

FIG. 3: Evolution of the cut-off of the random chain, as a function of the fraction of eliminated spins, under the two renormalization procedures discussed in the text. Note that the naive $M D H$ process generates interaction larger than those eliminated, even for strong disorder $(G=0)$. This never occurs in the new procedure used here.

We first consider the strong disorder case $G=0$. This corresponds to the quantum critical point of the phase diagram where the system flows to an infinite randomness fixed point. This becomes clear when we consider the fixed point form of the probability distribution of interactions. This is given by,

$$
P(J)=\frac{\alpha}{\Omega}\left(\frac{\Omega}{J}\right)^{1-\alpha}
$$

The exponent $\alpha$ as a function of the cut-off $\Omega$ is shown in Fig. 目. It varies as,

$$
\alpha=\frac{-1}{\ln \Omega}
$$

This behavior characterizes the strong disorder case, $G=$ 0 , as a random singlet phase [2].

Further evidence for a random singlet phase at $G=0$ is obtained considering the fraction of remaining active spins $\rho$ as a function of the energy scale set by the cut-off $\Omega$ [2]. This relation introduces a new exponent $\psi$ which is defined by,

$$
\rho=\frac{1}{L}=\frac{1}{|\ln \Omega|^{1 / \psi}} .
$$

It also establishes the connection between the characteristic length $L$ and the energy scale $\Omega$ for the case of logarithmic scaling. This is an extension of the usual definition of a dynamic exponent $\left(\Omega^{-1} \propto \tau \propto L^{z}\right)$. In Fig. 5 we show the density $\rho=1 / L$ as a function of the cut-off. From this expression we extract the exponent 


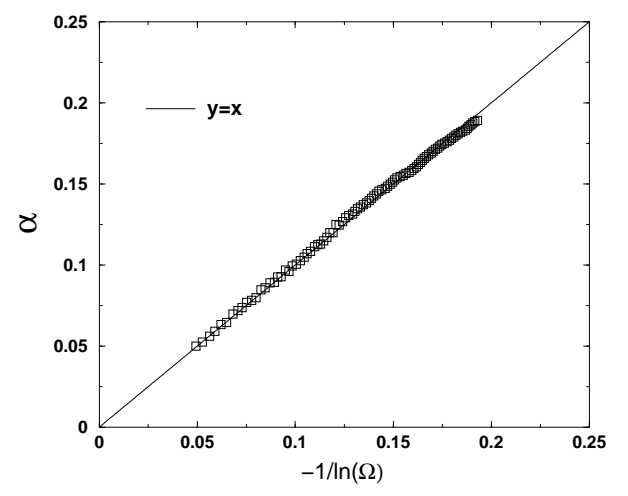

FIG. 4: Exponent $\alpha$ of the fixed point, power law distribution, Eq. 2, as a function of the cut-off $\Omega$ in the low energy limit.

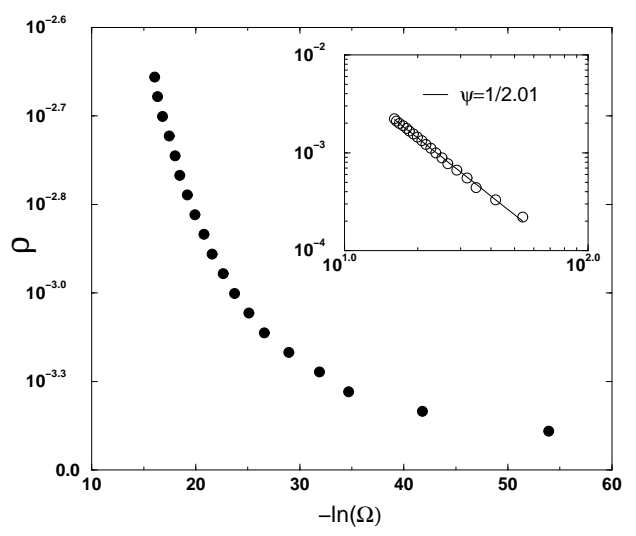

FIG. 5: The density of active spins as a function of the cut-off in the low energy limit. The inset shows the expected behavior for a random singlet phase with the exponent $\psi \approx$ $1 / 2$.

$\psi$ which takes the value $\psi=1 / 2$ characteristic of the random singlet phase [2].

Finally we calculate the distribution of first gaps at $G=0[7,8]$. This is obtained starting from a given configuration of random interactions for a chain of size $L$ and eliminating the spins, as described above, until a single pair remains. The interaction between these remaining spins yields the first gap $\Delta$ for excitation. Implementing this procedure for a large number of initial random configurations for chains of different sizes $L$ yields the distributions $P_{L}(\log \Delta)$ shown in Fig. 6. We considered over $10^{4}$ initial configurations to obtain the gap distributions. The widths of these distributions increase without limit as the sizes $L$ of the chains increase, as expected for an infinite randomness fixed point

According to the scaling form relating energy and length, Eq. A, we expect that the distribution $P\left(-\log \Delta / L^{\psi}\right)$ will present a universal behavior, independent of the size $L$ of the chains when plotted versus the variable $-\log \Delta / L^{\psi}$. This is indeed the case for
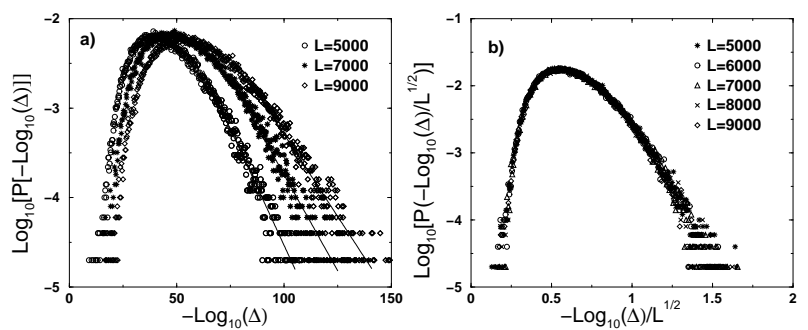

FIG. 6: a) Probability distribution of the first gap at the transition point, $G=0$. The distribution become broader and broader with L, which signals infinite randomness behavior. b) Scaling plot for the gap distributions. The collapse of the curves is obtained for $\psi=1 / 2$ as expected for a random singlet phase.

$G=0$ as shown in Fig. 6 for the $R S P$ exponent $\psi=1 / 2$.

We now decrease disorder increasing the gap in the exchange distribution. In Figure 7 we show the first gap distributions for different degrees of disorder as characterized by the gaps $G$ in the initial distribution of interactions. In all cases that we have investigated with $G \neq 0$, we find that the first gap distributions saturate at low energies in a form described by the expression, $P(\log \Delta) \sim \Delta^{1 / Z}$ for $\Delta \rightarrow 0$. The dynamic exponent $Z$ becomes independent of $L$ for $L$ sufficiently large. We have to consider large chains in order to observe this effect. We find $Z_{\infty} \sim 10.87, Z_{\infty} \sim 1.01$ and $Z_{\infty} \sim 0.68$ for $G=0.1, G=0.45$ and $G=0.5$, respectively. From these values of the dynamic exponent we can deduce the existence of a Griffiths phase extending up to $G_{G} \approx 0.45$ where the dynamic exponent reaches the value $Z=1$. For values of the gap $G>G_{G}$, i.e., small disorder, the dynamic exponent $Z<1$. The distribution of first gap for excitations, from which low temperature thermodynamic properties can be deduced, implies that $Z>1$ is required to obtain a singular behavior for these quantities with decreasing temperature. Consequently at $G_{G}$ there is a significant change in the nature of the thermodynamic behavior of the system. The phase for $G>G_{G}$ is a disordered Haldane phase with a pseudo-gap in the excitation spectrum. The thermodynamic behavior along the phase diagram will be explored in a future publication 22].

We have generalized the $M D H$ perturbative renormalization group. This method was known to fail in the case of random quantum chains with spins $S>1 / 2$ as it generates couplings which are larger than those eliminated, signalling the breakdown of perturbation theory. Taking into account larger clusters and treating them exactly we were able to circumvent this problem. For the important case of the spin-1 random Heisenberg antiferromagnetic chain our elimination procedure gives rise to interactions which are always smaller than those eliminated, even for weak disorder. This allows us to obtain the phase diagram of this system as a function of disorder. We find 

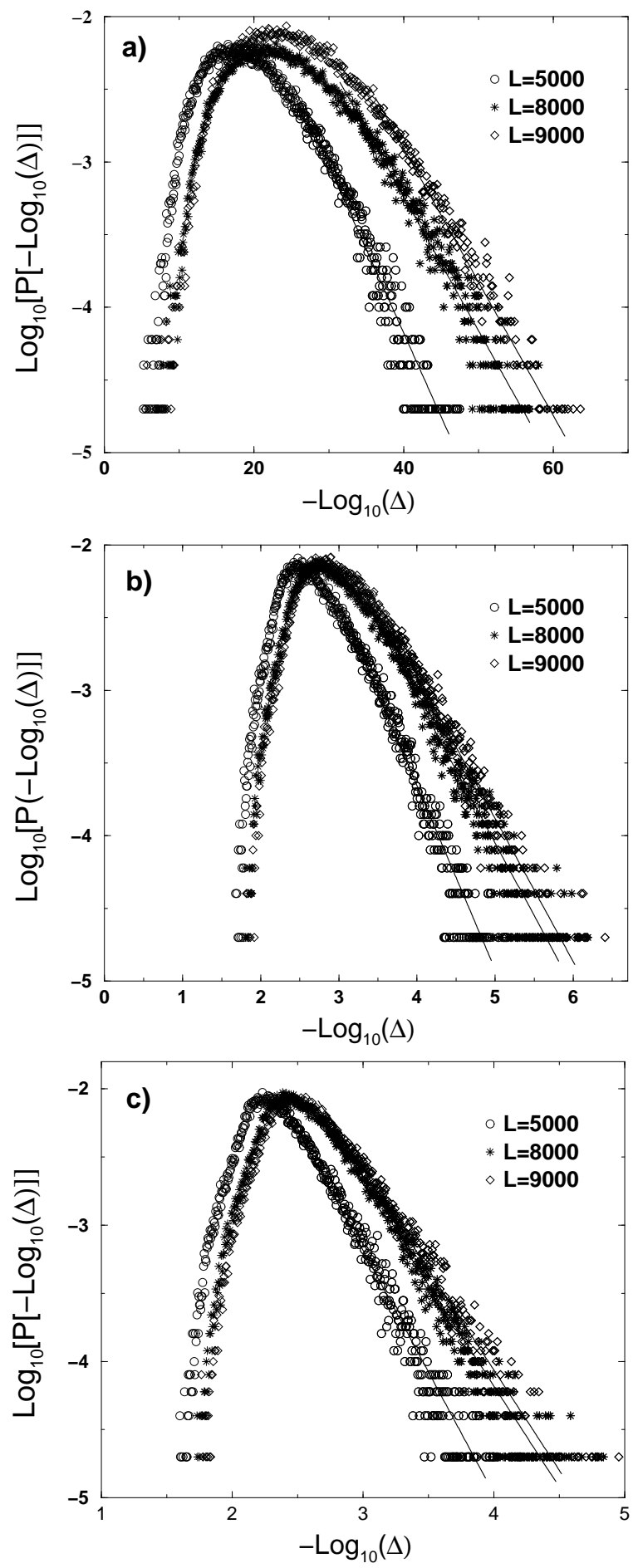

FIG. 7: Probability distributions of the first gap obtained from initial rectangular distributions of couplings with a gap $G$ and different systems sizes $L$. For clarity not all values of $L$ are shown. The solid lines represent best fits to the form $\log _{10}\left[P\left(-\log _{10} \Delta\right)\right]=A_{L}-\frac{1}{Z_{L}} \log _{10} \Delta$. a) $G=0.1$, $Z_{5000}=8.69, Z_{6000}=8.70, Z_{7000}=10.45, Z_{8000}=10.85$ and $Z_{9000}=10.87$. b) $G=0.45, Z_{5000}=0.79, Z_{6000}=0.81$, $Z_{7000}=0.96, Z_{8000}=1.0$ and $Z_{9000}=1.01$. c) $G=0.5$, $Z_{5000}=0.55, Z_{6000}=0.58, Z_{7000}=0.63, Z_{8000}=0.67$ and $Z_{9000}=0.68$.

for initial rectangular distributions with no gap, $G=0$, a random singlet phase similar to that found in spin $-1 / 2$ chains. For finite values of the gap $G$, the first gap distribution $P_{L}(\log \Delta)$ becomes, in the low energy limit, independent of $L$ for sufficiently large chains and is characterized by a dynamic exponent $Z$ which depends on how far the system is from the infinite randomness fixed point at $G=0$. This Griffiths phase is associated with a dynamic exponent $Z \geq 1$ and is limited by the fixed point at $G=0$ (where $Z=\infty$ ) and by $G_{G} \approx 0.45$, where $Z$ attains the value $Z=1$. For larger values of the gap, i.e., small disorder the system presents a disordered Haldane phase with a pseudogap in the spectrum for excitations.

We would like to thank Conselho Nacional de Desenvolvimento Científico e Tecnológico-CNPq-Brasil (PRONEX98/MCT-CNPq-0364.00/00), Fundacão de Amparo a Pesquisa do Estado do Rio de JaneiroFAPERJ for partial financial support.

* Electronic address: mucio@if.uff.br

[1] S.K. Ma, C. Dasgupta and C.K. Hu, Phys. Rev. Lett. 43, 1434 (1979); S.K. Ma, C. Dasgupta, Phys. Rev. B 22, 1305 (1980).

[2] D.S. Fisher, Phys. Rev. B 50, 3799 (1994); Phys. Rev. B 51, 6411 (1995).

[3] B. Boechat, A. Saguia e M.A. Continentino, Sol. St. Comm. 98, 411 (1996).

[4] M.A. Continentino, et al., Philos. Mag. B 73, 601 (1996).

[5] A. Saguia, B. Boechat and M.A. Continentino, Phys. Rev. B 58, 58 (1998); Phys. Rev. B 62, 5541 (2000).

[6] A. Saguia, B. Boechat and M.A. Continentino, Phys. Rev. B63, 52414 (2001).

[7] R. Mélin, Y.-C. Lin, P. Lajkó, H. Rieger, and F. Iglói, Phys. Rev. B 65, 104415 (2002).

[8] Y.-C. Lin, N. Kawashima, F. Iglói, and H. Rieger, Prog. Th. Phys. (Suppl.) 138, 470 (2000).

[9] F. Iglói, Phys. Rev. B 65, 064416 (2002).

[10] E. Carlon, P. Lajkó, and F. Iglói, Phys. Rev. Lett. 87, 277201 (2001).

[11] G. Refael. S. Kehrein, and D.S. Fisher, preprint cond-mat/0111295.

[12] Kedar Damle, preprint cond-mat/0201118.

[13] R.A. Hyman and Kun Yang, Phys. Rev. Lett. 78, 1783 (1997).

[14] C. Monthus, O. Golinelli, and Th. Jolicoeur, Phys. Rev. B 79, 3254 (1997); Phys. Rev. B 58, 805 (1998).

[15] K. Hida, Phys. Rev. B 45, 2207 (1992).

[16] K. Hida, Phys. Rev. Lett. 83, 3297 (1999).

[17] O. Motrunich, S.-C. Mau, D.A. Huse, and D.S. Fisher, Phys. Rev. B 61, 1160 (2000).

[18] see Quantum Scaling in Many-body Systems, M. A. Continentino (World Scientific, Singapore) 2001.

[19] see Field Theories of Condensed Matter Systems, E. Fradkin (Addison-Wesley Publishing Company, USA), 1994.

[20] F.D.M. Haldane, Phys. Lett. 93 A, 464 (1983).

[21] This is checked at every elimination.

[22] A. Saguia et al. to be submitted. 